\title{
Visualisation of Honeypot Data Using Graphviz and Afterglow
}

Craig Valli

Edith Cowan University

Follow this and additional works at: https://commons.erau.edu/jdfs|

Part of the Computer Engineering Commons, Computer Law Commons, Electrical and Computer Engineering Commons, Forensic Science and Technology Commons, and the Information Security Commons

\section{Recommended Citation}

Valli, Craig (2009) "Visualisation of Honeypot Data Using Graphviz and Afterglow," Journal of Digital Forensics, Security and Law. Vol. 4 : No. 2 , Article 2.

DOI: https://doi.org/10.15394/jdfsl.2009.1056

Available at: https://commons.erau.edu/jdfsl/vol4/iss2/2

This Article is brought to you for free and open access by the Journals at Scholarly Commons. It has been accepted for inclusion in Journal of Digital Forensics, Security and Law by an authorized administrator of Scholarly Commons. For more information, please contact commons@erau.edu. 


\title{
Visualisation of Honeypot Data Using Graphviz and Afterglow
}

\author{
Craig Valli \\ secau - Security Research Centre \\ Edith Cowan University \\ c.valli@ecu.edu.au
}

\begin{abstract}
This research in progress paper explores the use of Graphviz and Afterglow for the analysis of data emanating from a honeypot system. Honeypot systems gather a wide range of data that is often difficult to readily search for patterns and trends using conventional log file analysis techniques. The data from the honeypots has been statically extracted and processed through Afterglow scripts to produce inputs suitable for use by the DOT graph based tools contained within Graphviz. This paper explores some of the benefits and drawbacks of currently using this type of approach.
\end{abstract}

Keywords: honeypot, network forensics, visualization, Graphviz, Afterglow

\section{INTRODUCTION}

Honeypots generate a large amount of raw data for analysis and investigation by network security professionals. This raw data is typically rich in content and volume due to the modus operandi of network honeypots. This captured data can include but not be limited to system log files, network intrusion detection system log files, binary capture files and also malware candidate files.

On a relatively low interaction honeypot this data can run to several megabytes of textual and binary data per day. The analysis and trapping of malfeasance for which a honeypot system is designed is not an ideal match to traditional logfile analysis tools and techniques. Standard log file processors can only achieve so much in their ability to interpret the textual data that is captured by a honeypot into its log files. While log file processors have traditionally rich analysis algorithms they tend to display the top 10 or 100 in any selected investigated category or activity enabled by a suitable configuration of the underlying engine to ascertain this. While this modus operandi maybe a viable method for log file analysis based from a per use perspective such as world wide web access monitoring by business users it is typically not suited to analysis of malicious activity. This lack of viability is because often malicious activity by individuals and even the malicious code (malcode) they produce to 
enable an attack is performed or developed so as not to be detected. This posture of low detection is deleterious to how conventional log file analysis works.

Even dedicated intrusion detection query engines such as ACID, BASE or Surfnet IDS can have trouble locating relevant data within the mass of data that a honeypot will produce on a daily basis. The modus operandi of some attacks for instance may only utilize a single bot or compromised host for 1 or 2 interactions on a target. Some of the malcode will use the same attack vector typically the same port or initializing data stream but control 1000s of bots to cause a denial of service or overwhelm a host. Textually this is often hard for the human to see the associations and due to sometimes the high level of traffic this generates significant text is also generated.

The use of customized text scripts while a potentially optimal outcome for a particular scenario typically does not scale well in a live analysis scenario or is often not suitable for reuse. The exploration of alternative methods for interpreting and responding to honeypot data is necessary to progress the state of network security. Firewalls and other perimeter countermeasures are starting to show their age in the same way as the bastilles and castles of the middle ages similarly became in effective against new attacks. This paper is one such exploration of using Graphviz and associated log file processing scripts based around the AfterGlow suite to map certain types of honeypot data for interpretation by an investigator or researcher.

\section{WHAT IS GRAPHVIZ AND AFTERGLOW?}

Graphviz is an open source visualization utility developed to generate a variety of graph layouts (Ellson and Gansner 2008). There are several base utilities neato which makes "spring model" layouts, twopi which generates radial layouts and circo which generates circular layouts. They all interpret files that have been described using the DOT language. Afterglow is a series of PERLscripts designed to be used with Graphviz to generate link graphs from Comma Separated Values (CSV) formatted files (Marty 2007). The Afterglow conversion scripts provided take a raw input file (tcpdump, Snort, iptables and Argus logfiles), analyze it and output a comma separated list of records for each of the particular formats. The resulting records are based on the data present in the file and what the investigator would like represented from the complete record via configuration. Essentially the process is one of normalisation of the data into meaningful sets for analysis and subsequent graphical representation.

These customized scripts are not the only method of extraction of the records. Extraction can be accomplished manually using a spreadsheet program a slow and somewhat cumbersome event and also limited to processing 65535 or the row limit of the spreadsheet package in use. The use of spreadsheets is not suited to 
automatic generation of the graphs direct from the network interface or log file stream which is a long term aim of this research. The use of scripts could also process these interactions live into a database structure in similar fashion that occurs already with Surfnet IDS.

After being processed by the conversion scripts or processes the extracted CSV files are then fed through scripts that produce one of two formats. Either it generates a DOT attributed graph language file - the input required by the graphviz library - or it can generate input for the large graphing library (LGL) that is used extensively in bioinformatics. This experimentation was focused around the production of DOT attributed graphics for processing via Graphviz.

Afterglow accepts either 2 or 3 columns of raw input data to map. For network forensics the 3 column format is the most useful as it can be used to map interaction/events between two entities or attributes within a networked exchange. This operation is particularly useful in tracking or visualizing behaviors that are hard to interpret from text files or represent readily by other graphical or statistical methods.

\section{THE HONEYPOT SYSTEM}

A nepenthes honeypot system was used as the source data for this research (Baecher, Koetter et al. 2009). A nepenthes based honeypots purpose is to pose deceptively as a vulnerable target platform for malcode to interact with. It achieves this by the emulation of known vulnerability for example MS03-26 (Microsoft 2003) and other known security exploits that allow for upload of malcode or the execution of arbitrary code on the victim computer. Nepenthes via subsequent selective emulation of the known exploit to the malfeasant agent attempts to enable the successful download or transmission of malcode payload for subsequent capture and post incident analysis. Post incident analysis is normally the static and dynamic analysis of the malcode using specialized tools such as disassemblers.

Nepenthes logs and traps a wide range of data types and can store it in a wide range of formats for use by analysts. Nepenthes systems are also typically hardened and use firewalling to redirect or deny connections of interest to the honeypot system. The utilization of a system such as the Surfnet IDS system also extends the dataset that is available to the network forensics researcher. Surfnet not only logs data in a variety of formats but also processes captured malcode and runs it against virus scanners and sandboxes. It also allows aggregation of nepenthes data and external data from sandbox outputs from Anubis and CWSandbox for further analysis.

Table 1 is some example raw logged data from a nepenthes honeypot. The following data is extracted from the logged_downloads file that records when a piece of malware has been downloaded by nepenthes. 


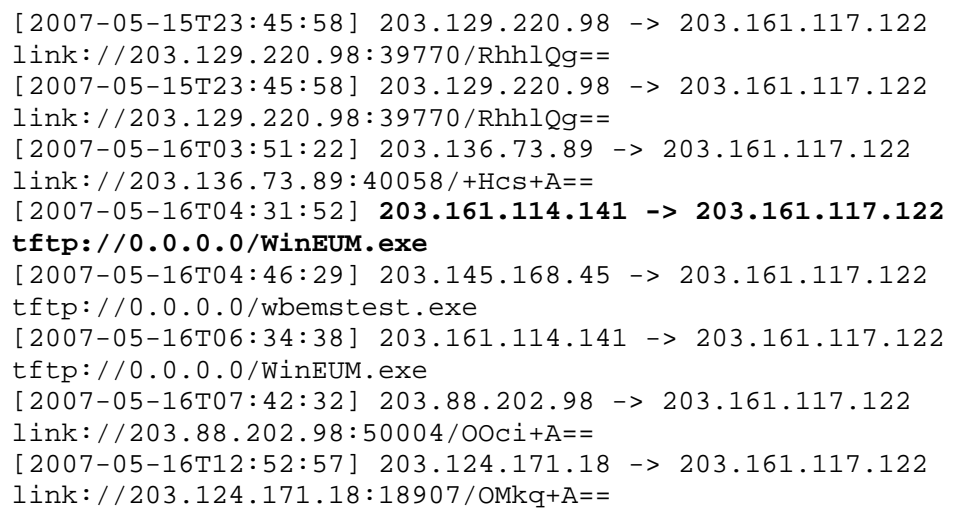

Table 1 - Raw data from nepenthes

Its basic format is Date \& Time expressed as YYYY-MM-DD (T) HH:MM:SS, Source IP, Destination IP, Protocol (Serving IP:Port)/PayloadName. This richness of record enables a wide amount of alternate lenses for analyzing data using the Graphviz engine for analysis. Table 2 is a partial representation of combinations as there are 24 possible combinations from this log file format excluding dates, inclusive of dates there would be 120 .

\begin{tabular}{|l|l|l|}
\hline Source IP & Destination IP & Payload \\
\hline 203.161.114.141 & 203.161 .117 .122 & WinEUM.exe \\
\hline Source IP & Destination IP & Protocol \\
\hline 203.161 .114 .141 & 203.161 .117 .122 & Tftp \\
\hline Source IP & Destination IP & PayloadIP \\
\hline 203.161.114.141 & 203.161 .117 .122 & 0.0 .0 .0 \\
\hline Source IP & PayloadIP & Destination IP \\
\hline 203.161.114.141 & 0.0 .0 .0 & 203.161 .117 .122 \\
\hline Payload & Source IP & Destination IP \\
\hline WinEUM.exe & 203.161 .114 .141 & 203.161 .117 .122 \\
\hline Protocol & Source IP & PayloadIP \\
\hline Tftp & 203.161 .114 .141 & 0.0 .0 .0 \\
\hline
\end{tabular}

Table 2 - Some combinations from logfile data

Some of the combinations would clearly not be useful for analysis and other would produce very similar graphical representations of the data. The remaining meaningful combinations in turn do give a different view of the data in visualized form when compared to static graphics and textual outputs of conventional analysis tools. A selection of these are displayed on the following figures.

It can be clearly seen in Figures 1 through 4 that the same data set can have many 
different visualizations or lenses for analysis of the dataset. Each of the visualizations tells a slightly different story by the lense or representation that it provides the investigator into the raw data set. The ability to use different lenses allows an investigator to view data rapidly and from different viewpoints that can significantly aid in interpretation, detection and potential amelioration of issues or events. This potential for rapid interpretation that a graphical data view provides is simply not readily achievable through text based analysis. It is easy to see different holistic patterns in the presented data even though in each figure they are drawn from the same dataset, they just have a different articulation or causal linkage when they have been interpreted and represented by the Graphviz utilities.

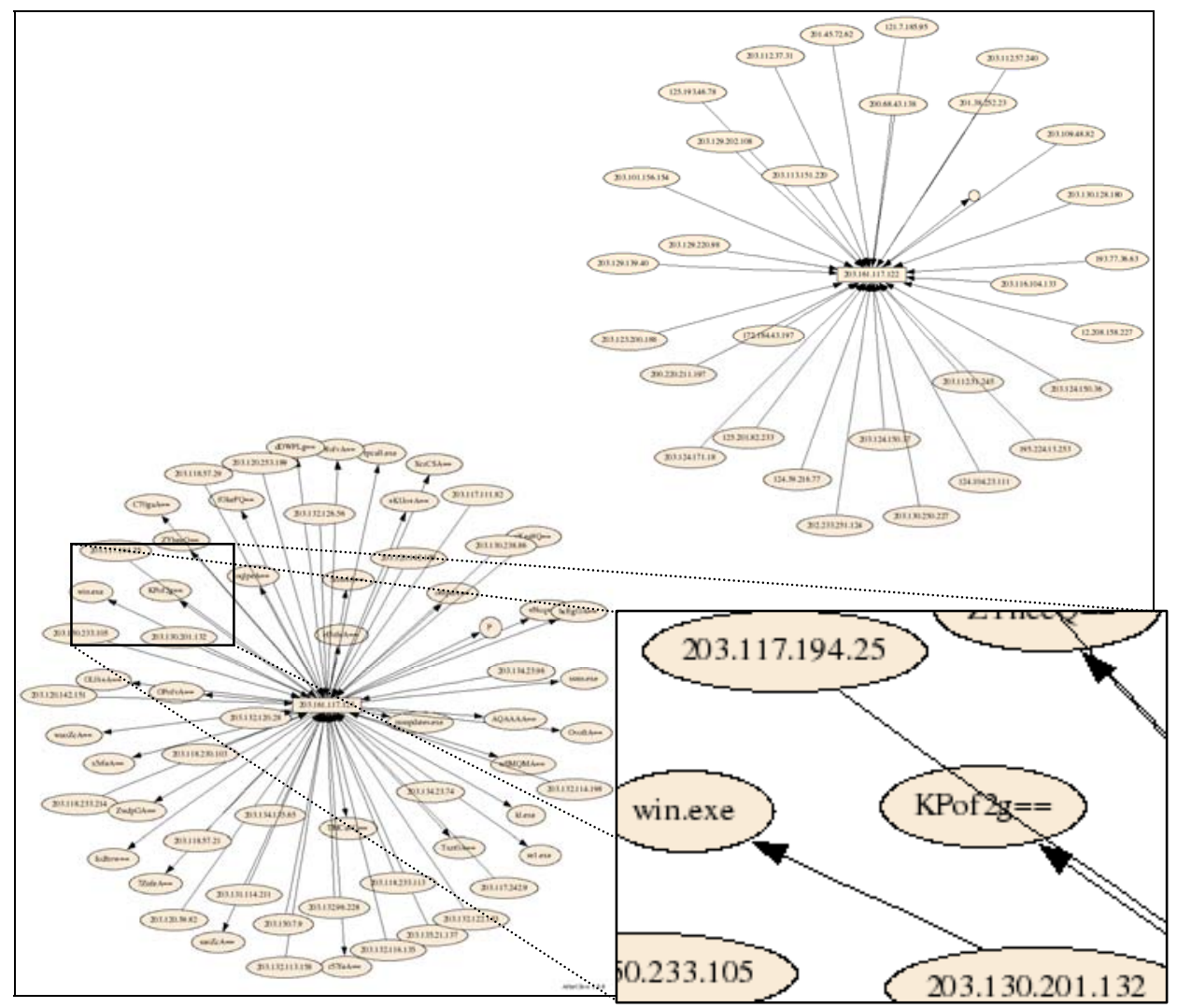

Figure 1. SIP PIP Payload 
Journal of Digital Forensics, Security and Law, Vol. 4(2)

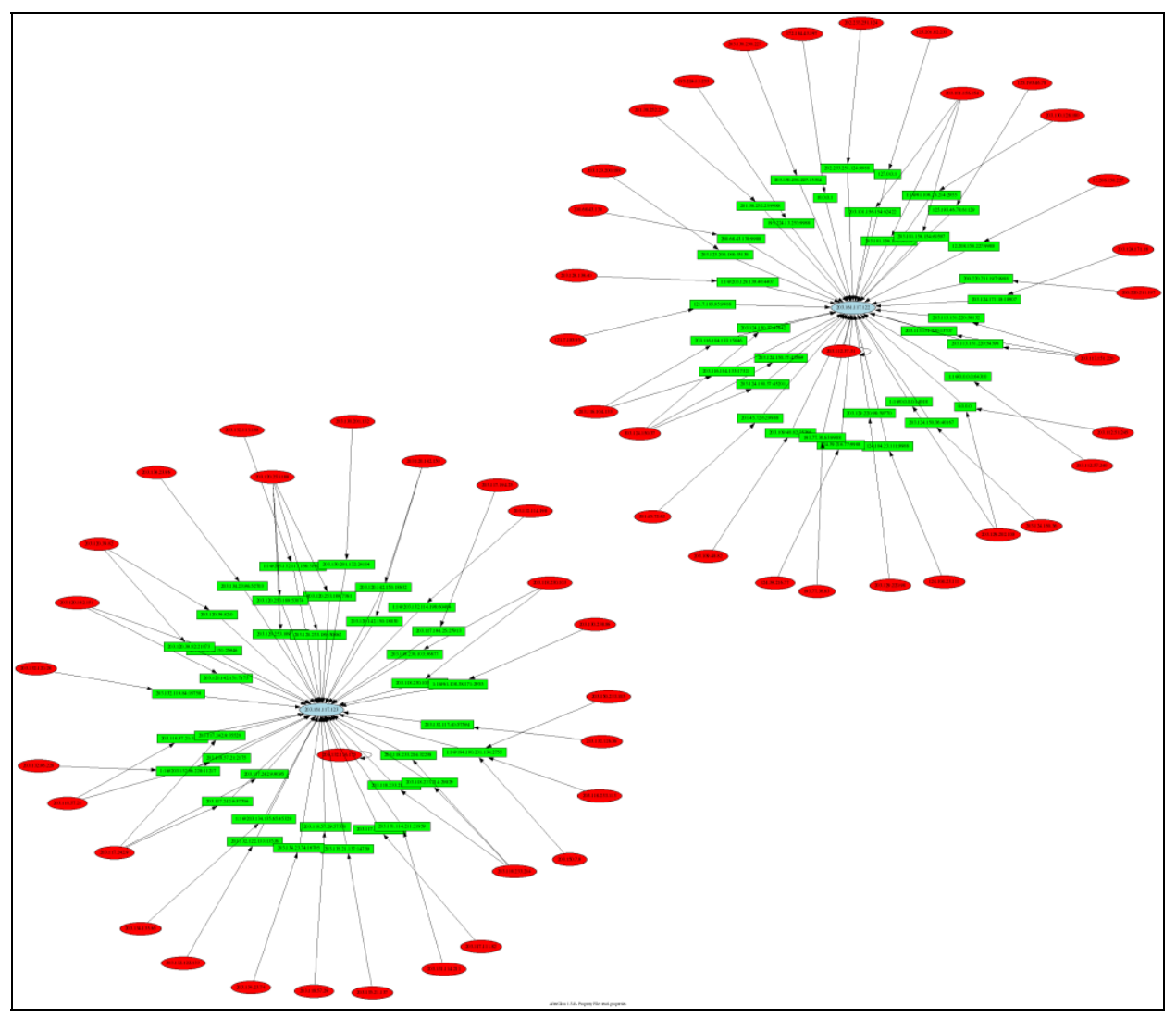

Figure 2. SIP PIP DIP 


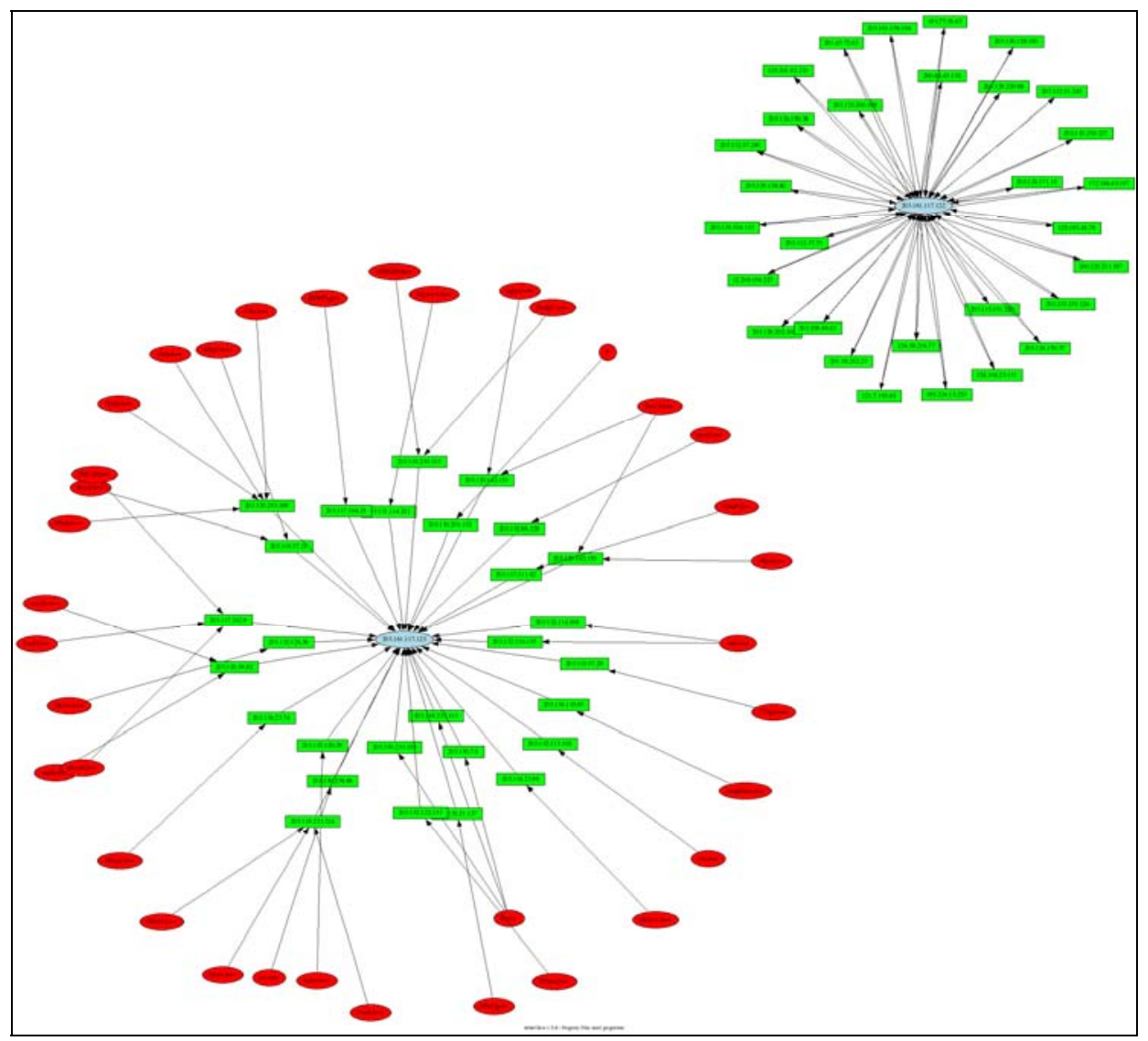

Figure 3. Payload SIP DIP 
Journal of Digital Forensics, Security and Law, Vol. 4(2)

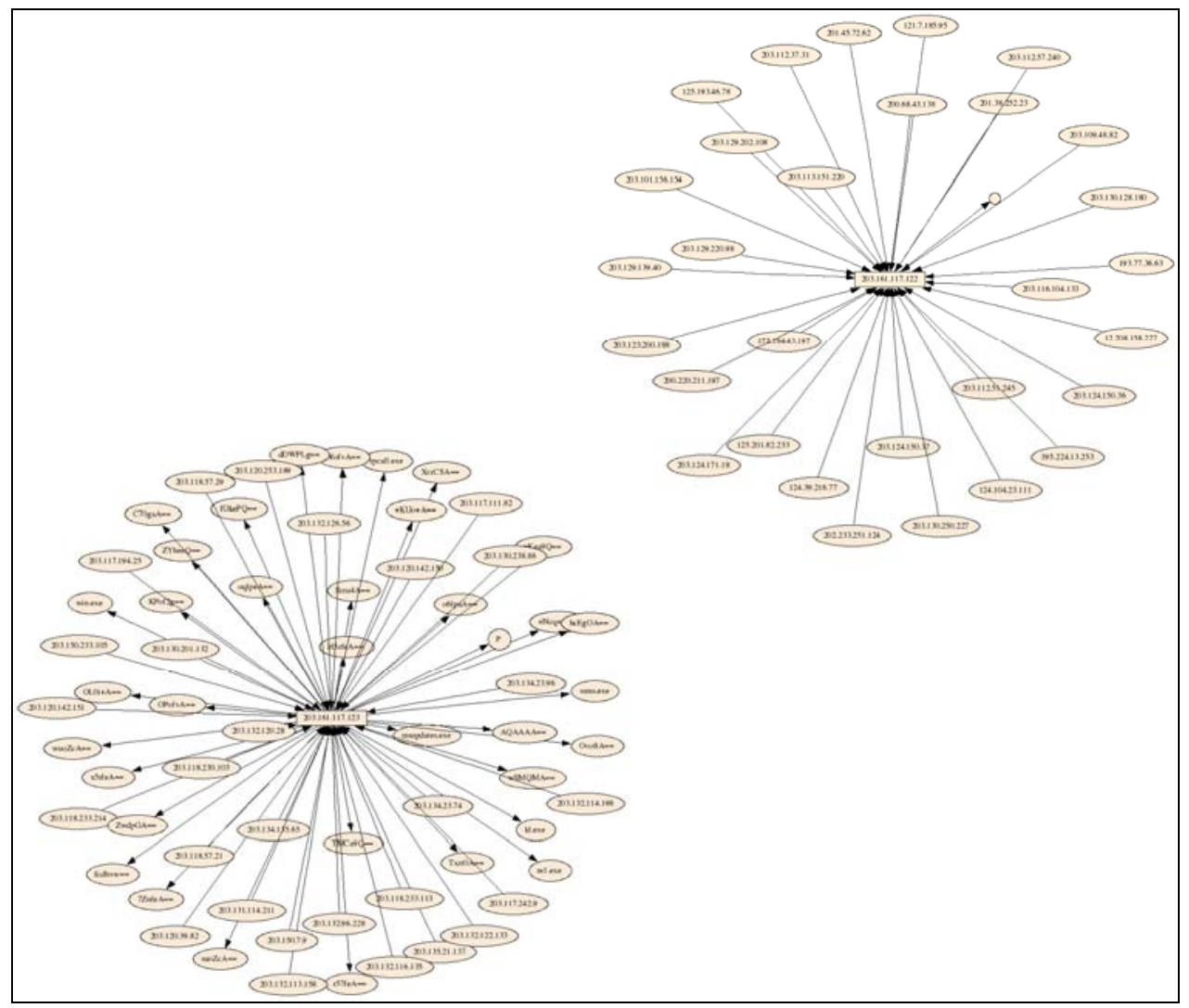

Figure 4. SIP PIP Payload 
The graphs represented here are by no means the largest generated in this initial research. One graph that used the complete set of data generated a 35000 x 33000 pixel bitmap file. Even with this large file it was clear to see from a holistic perspective the epicenters or areas of high network activity. Figure 5 is a very reduced snapshot of that file.

The graphical nature of the method as clearly demonstrated in Figure 5 demonstrates it is relatively easy to pick out areas of concentration of attack. This method elucidates how also this type of graphical representation performs clustering and subsequent visualization of like events experienced on honeypot systems. These groupings unlike conventional methods of interpretation do not suffer from the same temporal separation that other log file analysis tools can have for instance if these attacks were perpetrated across a range of weeks. The focus for the generation of the graphics is the entity, with the size of the ring or circle in this case indicating magnitude and depth of attack. It is far easier to track extended attacks using these methods of analysis and presentation than conventional log file analysis techniques due to the visual clustering of the data representations.

The graphical nature of these types of outputs provides a medium for the rapid interpretation of patterns and emergent trends. The use of DOT graphs generated from Graphviz provides a method for making a particular entity or entities the focal point of the analysis in a consistent visual manner for the human analyst. 


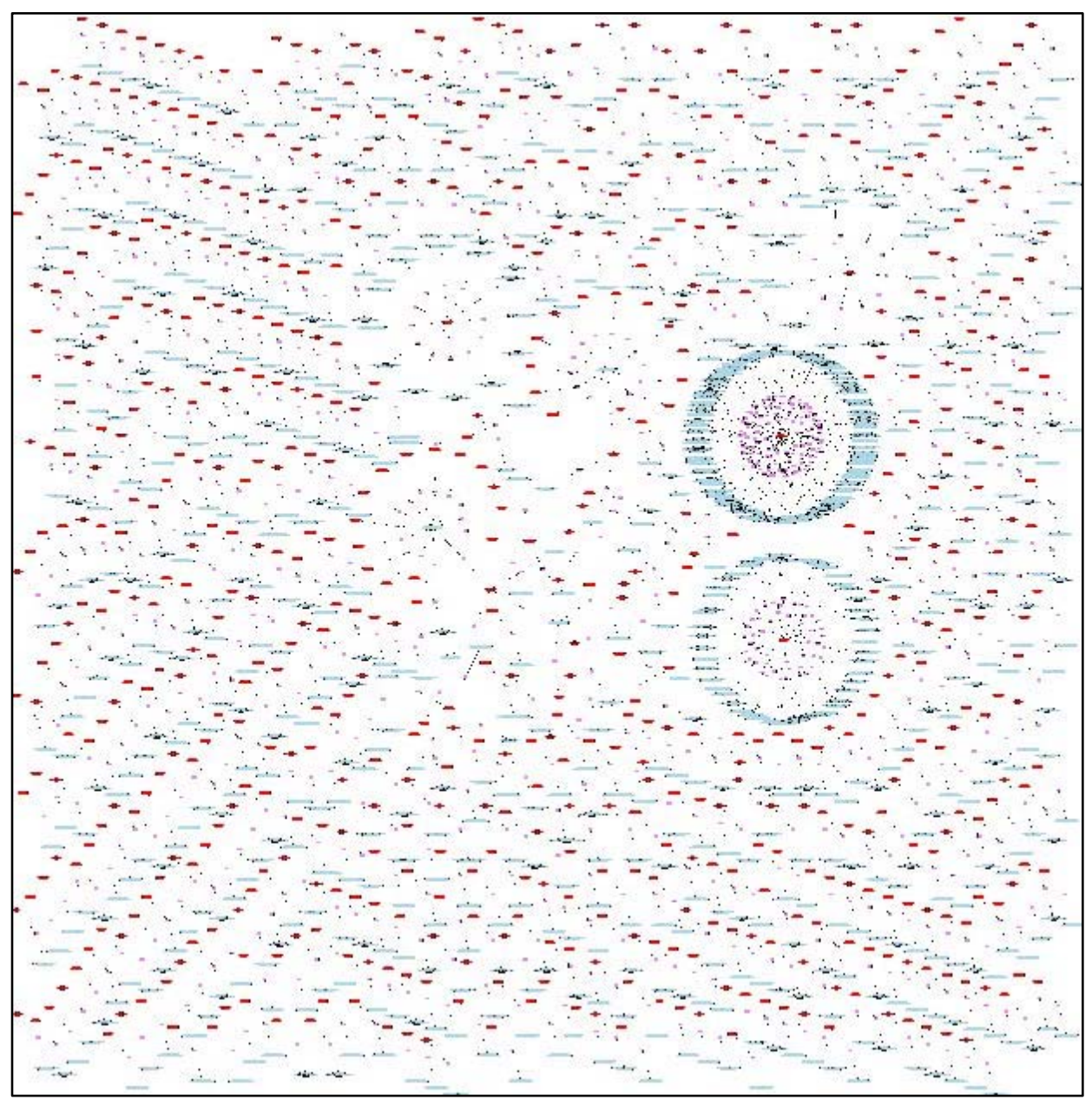

Figure 5. Reduced snapshot of large visualisation 


\section{CURRENT DRAWBACKS AND LIMITATIONS}

Currently the generation of bitmap Graphviz dot graphs is a processor intensive task. The author used a 64 bit Debian based Dual Core - Dual Opteron $2.8 \mathrm{GHz}$ machine with 6GB of memory with a U320 SCSI hard disk subsystem during the development of graphs for this research. To generate even the relatively simple sample graphs contained in Figures 1 to 4, considerable runtime at 100\% of CPU was experienced for several minutes on the experimental computer.

This current use of computing resource has significant impact on the ability to use this type of technology currently for generation of near real time graphical data from a honeypot, IDS, firewall or similar traditional network countermeasure. The amount of data processed in the initial trials was comparatively small compared to the level of incident that would need to be rendered on a larger network pipe or dataset.

These particular problems of computational shortfall maybe overcome by utilization of current GPU technology to render and also compute the graphics (Ellson and Gansner 2008). Similar use of GPUs has been recently successfully exploited for rapid visualization of graphical data where combining 4 high end GPU cards has produced graphical computational performance equivalent to 320 2.4Ghz CPU cores (ASTRA 2008). The application of GPU technology to certain mathematical functions and graphical rendering tasks delivers teraflops of performance at a relatively low cost. This type of power yield is dependent on the types of mathematical functions involved in the processing, however it would appear that Graphviz and its utilities may significantly benefit from the utilization of this technology.

The incorporation of a SQL system to store and process data extracted from honeypot data streams and instead of reprocessing log files will be a significant leap forward in analysis turnaround. Interfacing with the Surfnet IDS system that incorporates nepenthes data into an existing SQL structure is an easy candidate system for supporting SQL capabilities with a Graphviz/afterglow engine for generation of graphics. This extension of Surfnet would enable not only near real time generation of graphical data but also provide a method for longitudinal analysis of patterns and trends relating to honeypot activity. As mentioned previously this type of system reduces some of the temporal and spatial issues that are found in conventional textual query and analysis engines. The system will also allow replaying of items of interest by extracting it from the database. Temporary storage within a database structure would also allow for some buffering and load balancing to occur, by smoothing out spikes in the transmissions received.

\section{CONCLUSION}

This is research in progress, however, there are clear benefits for the analyst in 
using graphical approaches to perform analysis of honeypot data. Particularly as virus scanners, firewalls and intrusion detection systems are starting to fail as a response and countermeasure and honeypots are now trapping malcodes that bypass these with relative impunity.

Currently there is a bottleneck with regards to fast processing of data that would be arriving on a network interface in a honeypot system. The use of modern GPU technology and high-speed storage technologies may see many of these impediments removed. Also there are methods of the vector based rendering of the images which maybe better suited to interpretation of live data. These will be explored in the next phase of this research.

The ability to use graphical representations to view data from a variety of viewpoints and lenses for near real-time forensics of network incidents is a compelling reason for furthering this type of research and investigation. The graphical methods utilized in this exploratory work demonstrate an ability to represent magnitude and intensity of attack not afforded to text based analysis of data. By using graphical techniques such as these it may yield significant benefit in detecting and ameliorating threats that are now posed by network borne malcodes which are increasingly becoming a problem in the network security arena.

\section{REFERENCES}

ASTRA. (2008). "Belgian researchers develop desktop supercomputer.", from http://fastra.ua.ac.be/en/index.html.

Baecher, P., M. Koetter, et al. (2009). "nepenthes." from

http://nepenthes.carnivore.it/.

Ellson, J. and E. Gansner (2008). Graphviz - Graph Visualization Software, AT\&T.

Marty, R. (2007). "Afterglow." from http://afterglow.sourceforge.net.

Microsoft. (2003). "Microsoft Security Bulletin MS03-026 - Buffer Overrun In RPC Interface Could Allow Code Execution (823980)." Retrieved 6th Feb, 2006, from

http://www.microsoft.com/technet/security/bulletin/MS03-026.mspx. 